\title{
Magnetic properties of alternating Hubbard ladders
}

\author{
Kaouther Essalah and Ali Benali \\ Department of Physics, Faculty of Sciences of Tunis, \\ University of Tunis El-Manar, Tunis 2092, Tunisia \\ Anas Abdelwahab and Eric Jeckelmann \\ Leibniz Universität Hannover, Institut für Theoretische Physik, Appelstr. 2, 30167 Hannover, Germany \\ Richard T. Scalettar \\ Department of Physics, One Shields Ave., University of California, Davis, California 95616, USA
}

(Dated: April 14, 2021)

\begin{abstract}
We investigate the Hubbard Hamiltonian on ladders where the number of sites per rung alternates between two and three. These geometries are bipartite with non-equal or equal number of sites on the two sublattices. Thus they share a key feature of the Hubbard model in a class of lattices which Lieb has shown analytically to exhibit long-range ferrimagnetic order, while being amenable to powerful numeric approaches developed for quasi-one-dimensional geometries. The Density Matrix Renormalization Group (DMRG) method is used to obtain the ground state properties, e.g. excitation gaps, charge and spin densities as well as their correlation functions at half-filling. We show the existence of long-range ferrimagnetic order in the one-dimensional ladder geometries. Our work provides detailed quantitative results which complement the general theorem of Lieb for generalized bipartite lattices. It also addresses the issue of how the alternation between quasi-long range order and spin liquid behavior for uniform ladders with odd and even numbers of legs might be affected by a regular alternation pattern.
\end{abstract}

\section{INTRODUCTION}

Artificially constructed quantum nanostructures of correlated electrons in quasi one (1D) and two (2D) dimensions can be described using lattice models such as Heisenberg and Hubbard Hamiltonians, as well as their extensions. Novel experimental realizations of such systems include the fabrication of arrays of magnetic atoms on surfaces using scanning tunneling microscopy [1-5], as well as optical lattice emulators using ultracold atoms [6-8]. Bipartite lattices constitute a particularly important class of geometries in which the nearestneighbor hopping (or magnetic exchange) is such that the lattice has two subsystems $A$ and $B$ in which only $B$ sites are nearest-neighbor of $A$ sites, and vice-versa. E. Lieb proved [9] a rigorous theorem for the Hubbard model on a bipartite lattice at half-filling: the ground state total spin quantum number is given by $S=\left(N_{B}-N_{A}\right) / 2$, where $N_{A}\left(N_{B}\right)$ is the number of sites in the $A(B)$ subsystem. Subsequently, Shen et al. [10] proved that both ferromagnetic and antiferromagnetic long-range orders coexist in the degenerate ground state with $S \neq 0$, i.e. the system exhibits long-range ferrimagnetic order. Subsequent work examining Lieb's theorem has mostly focused on 2D lattices such as Lieb's original " $\mathrm{CuO}_{2}$ " lattice.

On the other hand, during the past several decades, strongly correlated electron materials with quasi-onedimensional (1D) ladder structures [11] have attracted much attention theoretically as well as experimentally. These ladder structures reveal interesting phases including Luttinger liquids [12], Mott insulators [13], antiferromagnetism [14], as well as charge density waves
[15]. Early theoretical studies of Heisenberg ladders with only nearest neighbor interactions and without frustration revealed an interesting effect by changing the number of legs [12]. Geometries with an even number of legs are associated with a singlet ground state with a spin gap to the lowest-lying excitations and short-range spin correlations. For odd numbers of legs the ground state has quasi-long-range antiferromagnetic order and gapless spin excitations. Lattices with an odd number of legs are in the universality class of the single leg spin- $\frac{1}{2}$ Heisenberg chain [12].

Hubbard ladders at half-filling (one electron per site) reveal similar behavior for the spin excitation modes, while the charge excitation modes remain gapped [16]. This similarity is expected since there is a mapping between the Heisenberg model and the spin sector of the Hubbard model at strong values of the on-site electron repulsion $U$. The density matrix renormalization group (DMRG) [17-19] has proven to be an especially powerful computational tool in uncovering this physics.

In this article, we introduce novel ladder configurations which consist of an alternation of rungs with even and odd numbers of sites, and study the strong correlation physics of the Hubbard model. This structure serves as an intermediary between the even rung, gapped ladder, and the odd rung case which supports spin correlations with a power law decay. The magnetic properties are discussed in the light of Lieb's theorem and the imbalanced sublattice site count induced by the combination of an even and odd numbers of legs. Understanding the properties of these quasi-1D lattice geometries offers the opportunity to further understand, and potentially extend, Lieb's theorem. 


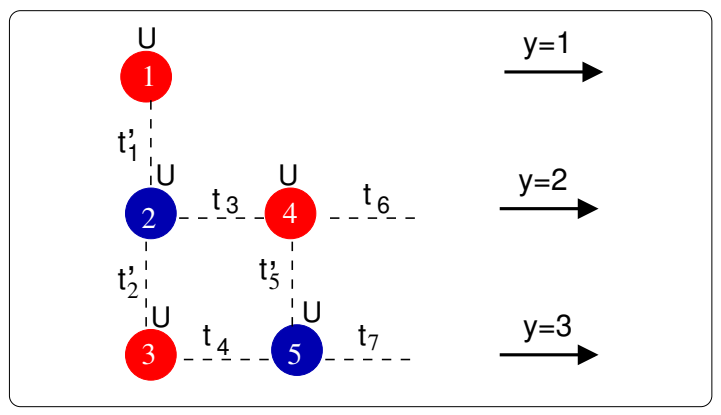

FIG. 1. 3-2 ladder geometry with alternating rung site numbers. The red and blue circles indicates the sites belonging to the sublattices A and B, respectively. Dashed lines label the non-zero hopping terms.

The paper is organized as follows. In the next section we discuss non-interacting nearest-neighbor tightbinding models on alternating ladder geometries, and obtain their band structure and density of states (DOS), which are crucial starting points for consideration of the effects of electronic correlations. With this groundwork, in section III, we use the DMRG method to investigate correlated Hubbard ladder systems with two different alternation frequencies. One pattern has $N_{A}=N_{B}$ and the other has $N_{A} \neq N_{B}$. Consideration of both cases allows us to isolate the effects of imbalanced sublattice site counts from that of alternating number of sites per rung. In the last section we summarize and discuss the main results of this work. The Appendices contain calculations for uniform 3-leg ladders, to facilitate comparison with our studies of alternating ladders, and some further details for the noninteracting alternating ladders.

\section{NONINTERACTING ALTERNATING LADDER GEOMETRIES}

In this section we derive the non-interacting dispersion relations and DOS of two, hitherto unstudied, geometries with alternating rung length. We investigate two alternation periods. The first has period $d=2$ in the leg direction: Each unit cell contains three sites in one rung and two sites in the second rung. We call this geometry the 3-2 ladder. It is bipartite with sublattices $A$ and $B$, but of the class that Lieb studied, with $N_{A} \neq N_{B}$. The second structure has period $d=4$. Each unit cell contains three sites on the first and second rungs followed by two sites in the third and fourth rungs. We call this geometry the 3-3-2-2 ladder. It has $N_{A}=N_{B}$. Appendix A reviews the band structure of uniform 3-leg ladders.

The nearest-neighbor tight-binding model is described

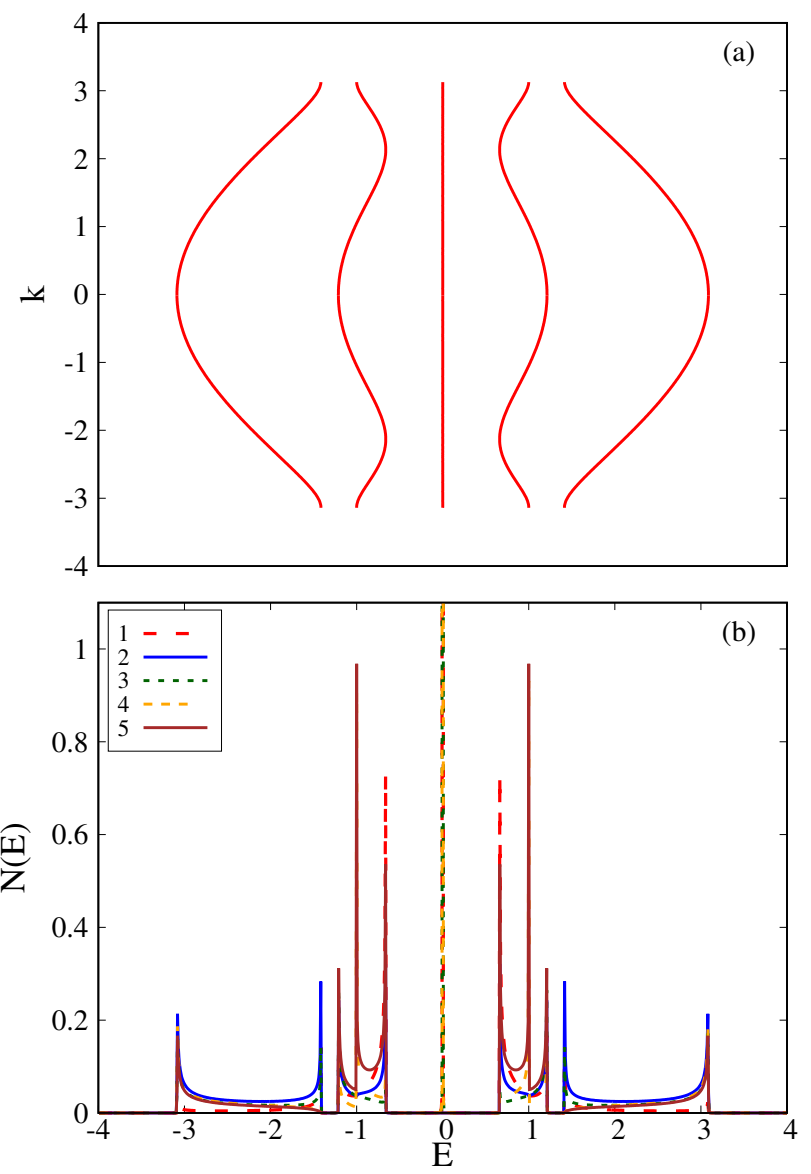

FIG. 2. (a) Band structure for the 3-2 alternating ladder in the noninteracting case for uniform hopping terms $t_{i}=t=$ 1. (b) Corresponding local density of states (3) for each site $j=1, \ldots, 5$ of the unit cell shown in Fig. 1 .

by the Hamiltonian

$$
\begin{aligned}
H_{0} & =-\sum_{x, y, \sigma} t_{x, y}\left(c_{x+1, y, \sigma}^{\dagger} c_{x, y, \sigma}+c_{x, y, \sigma}^{\dagger} c_{x+1, y, \sigma}\right) \\
& -\sum_{x, y, \sigma} t_{x, y}^{\prime}\left(c_{x, y+1, \sigma}^{\dagger} c_{x, y, \sigma}+c_{x, y, \sigma}^{\dagger} c_{x, y+1, \sigma}\right) .
\end{aligned}
$$

$c_{x, y, \sigma}^{\dagger}\left(c_{x, y, \sigma}\right)$ denotes the creation (annihilation) operators for an electron with spin $\sigma$ on the site with coordinates $(x, y)$ where $y=1,2,3$ denotes the Hubbard leg and $x=1, \ldots, L_{x}$ refers to the rung index. The parameters $t_{x, y}$ and $t_{x, y}^{\prime}$ are hopping amplitudes along and between the chains, respectively. They depend on the ladder geometry considered.

\section{A. The alternating 3-2 ladder}

The 3-2 ladder system is sketched in Fig. 1. Defining $L_{x}$ as the total number of rungs, the total numbers of sites on the two sublattices are unequal, with $N_{A}=$ $3 L_{x} / 2$ and $N_{B}=L_{x}$. 


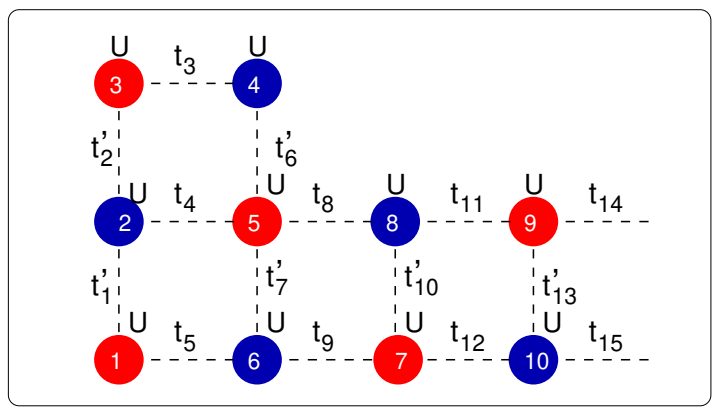

FIG. 3. Geometry of the alternating 3-3-2-2 ladder lattice. The red and blue circles indicate the sites belonging to the $A$ and $B$ sublattice, respectively. Dashed lines show the nonzero hopping terms.

$H_{0}$ can be written as a sum of commuting operators $H_{k}$ acting only on the Bloch states with wave number $k$ in the first Brillouin zone (see Appendix A to get the concrete form of wave number $k$ ). In the present case, we have five sites per unit cell, labeled $j=1, \ldots, 5$ in Fig. 1 . $H_{k}$ is the $5 \times 5$ matrix,

$$
H_{k}=\left(\begin{array}{ccccc}
0 & -t_{1} & 0 & 0 & 0 \\
-t_{1} & 0 & -t_{2} & -t_{3}-\widetilde{t}_{6} & 0 \\
0 & -t_{2} & 0 & 0 & -t_{4}-\widetilde{t}_{7} \\
0 & -t_{3}-\widetilde{t}_{6}^{*} & 0 & 0 & -t_{5} \\
0 & 0 & -t_{4}-\widetilde{t}_{7}^{*} & -t_{5} & 0
\end{array}\right)
$$

Here $\widetilde{t}_{j}=t_{j} \exp (i k)$.

We will investigate the system with equal hopping parameters $t_{i}=t$ for $i=1, \ldots, 7$, since this simple choice already contains several novel features. The diagonalization of $H_{k}$ leads to the five energy bands $E_{k, b}$ $(b=1, \ldots, 5)$ shown in Fig. 2(a). One of these bands is flat and is located at zero energy (the Fermi level for halffilling), in accordance with Lieb's theorem [9]. For such a flat band, there are gapless single-electron excitations but the system is not metallic in the sense that the effective mass of the low-lying excitations, which is proportional to $1 / t$ for a linear chain, is infinite. Figure 2(b) shows the corresponding local DOS

$$
N(E, j)=\frac{1}{N_{c}} \sum_{k, b}\left|\psi_{k, b}(j)\right|^{2} \delta\left(E-E_{k, b}\right)
$$

where $\psi_{k, b}(j)$ represent the eigenvectors of Eq. (2) corresponding to the eigenenergy $E_{k, b}, j=1, \ldots, 5$ are the sites of the unit cell. $N_{c}=L_{x} / 2$ is the number of unit cells (or equivalently the number of wave vectors $k$ in the first Brillouin zone). Note that all DOS distributions in this paper are normalized so that the integral over all energies $E$ is equal to 1 . To draw the DOS we substitute a Lorentzian function of width $\eta=0.01 t$ for the Dirac $\delta$-function. The singularity at $E=0$ in the local DOS is due to the flat band. Figure 2(b) reveals that this flat band is located on the $A$ subsystem, ie. sites 1, 3 and 4 in the unit cell in Fig. 1.

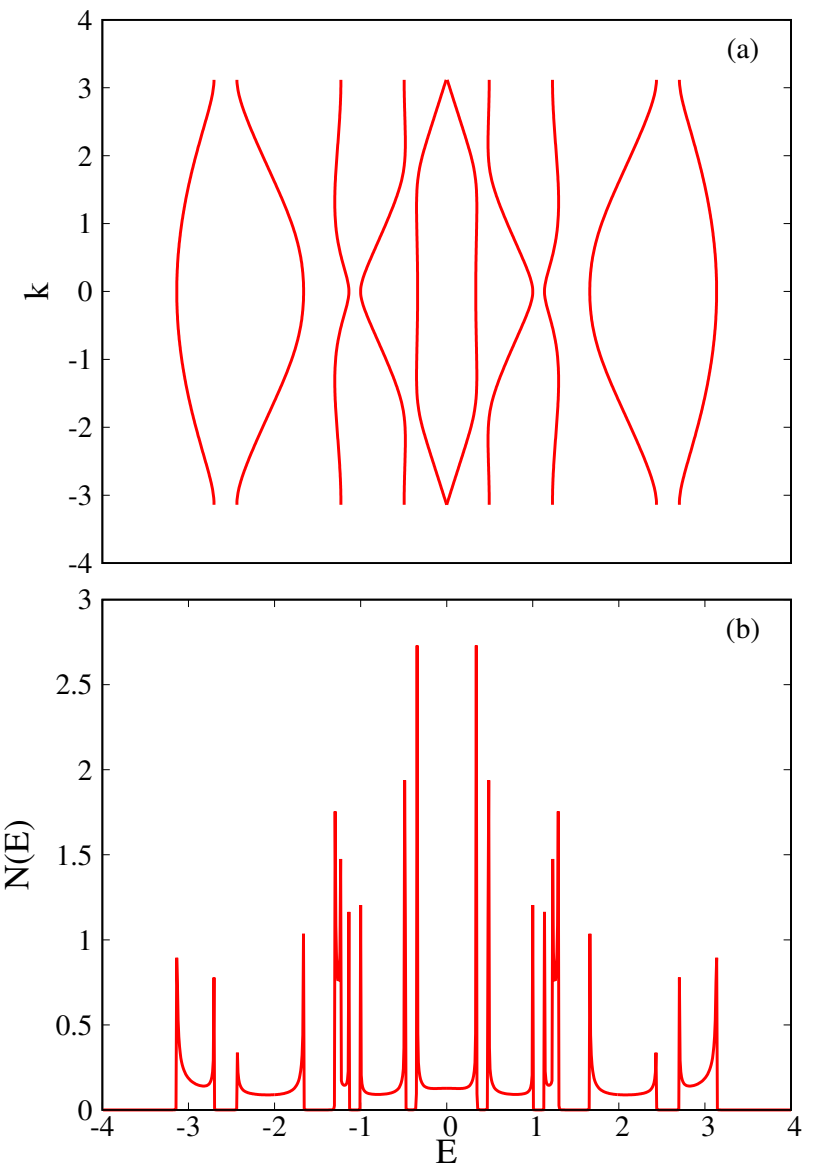

FIG. 4. (a) Band structure and (b) DOS, Eq.4, of the noninteracting alternating 3-3-2-2 ladder. Compared to the 3-2 geometry in Fig.2 the key difference is the absence of the flat band at $E=0$.

\section{B. Alternating 3-3-2-2 ladder}

The real space 3-3-2-2 ladder geometry is displayed in Fig. 3. The lattice is bipartite, with an equal number of sites in the sublattices $A$ and $B$. Similarly to the 3-2 ladder geometry, $H_{0}$ can be written as a sum of commuting operators $H_{k}$ acting only on the Bloch states with wave number $k$. Here there are 10 sites per unit cell. Its single-particle matrix representation $H_{k}$ is the $10 \times 10$ matrix given in Appendix B.

The diagonalization of the matrices

$H_{k}$ leads to the ten energy bands $E_{k, b}$ shown in Fig. 4(a). Since $N_{A}=N_{B}$, we do not observe a flat band. The system is metallic at half filling as the Fermi energy $E_{F}=0$ lies at a point where two bands touch. This is also visible in the total DOS

$$
N(E)=\frac{1}{N_{s}} \sum_{k, b} \delta\left(E-E_{k, b}\right)
$$

with division by the number of sites $N_{s}$ providing the previously described normalization convention for $N(E)$. 
This DOS is plotted in Fig. 4(b), which shows a finite density but no concentration of spectral weight at the Fermi level, in contrast to the peak seen in Fig. 2(b).

Although the 3-2 and 3-3-2-2 geometries share an alternation of number of sites in different rungs, they differ significantly due to the presence of a flat band in the 3-2 case. We will analyze in the next section how this difference is reflected in the properties of ladders with the on-site interaction turned on.

\section{DMRG RESULTS FOR ALTERNATING LADDERS}

In this section we analyze and compare the properties of the 3-2 and 3-3-2-2 ladder geometries in the presence of the Hubbard interaction using the finite-size DMRG method. The full Hamiltonian is

$$
H=H_{0}+U \sum_{x, y} n_{x, y, \uparrow} n_{x, y, \downarrow}
$$

with $n_{x, y, \sigma}=c_{x, y, \sigma}^{\dagger} c_{x, y, \sigma}$.

DMRG is widely considered to be the most powerful numerical method for quasi-1D correlated electron systems [20]. The details of this method have been reviewed in Ref. [21]. In our DMRG calculations, open boundary conditions are applied in both $x$ - and $y$ directions. Our program uses the conservation of the particle numbers $N_{\sigma}$ but not the $\mathrm{SU}(2)$ spin symmetry. The number $m$ of density-matrix eigenstates in the renormalization procedure is increased progressively until it reaches $m=2500$ in the last sweep. The total number of used sweeps is up to 13 . The discarded densitymatrix weight (truncation error) varies from $10^{-5}$ to less than $10^{-9}$. We extrapolate the ground state energy to the vanishing discarded weight limit [22] and estimate the error from the difference between the extrapolated energy and the energy in the last sweep. The finitesize error is obtained by varying the system length and extrapolating to vanishing ratio $1 / L_{x}$. In our calculations the ladder length $L_{x}$ is varied up to 200 . We have implemented the one-dimensional DMRG path through all lattice sites of the alternating ladder geometries so that the sites are ordered as in the standard approach for homogeneous ladders [21]. The ground state energy, spin, charge and single-particle gaps, the pair-binding energies, correlation functions, as well as spin and charge densities are investigated for a wide range of parameters. We use homogeneous hopping parameters $t_{i}=1$ everywhere.

\section{A. 3-2 alternating rung geometry}

According to Ref. [9], we expect the ground state at half filling to be ferromagnetic, with total spin $S=$ $\left(N_{A}-N_{B}\right) / 2=L_{x} / 4$ for any finite value of the on-site interaction $U>0$. We have first computed the eigenstate with the lowest energy as a function of the $z$-projection

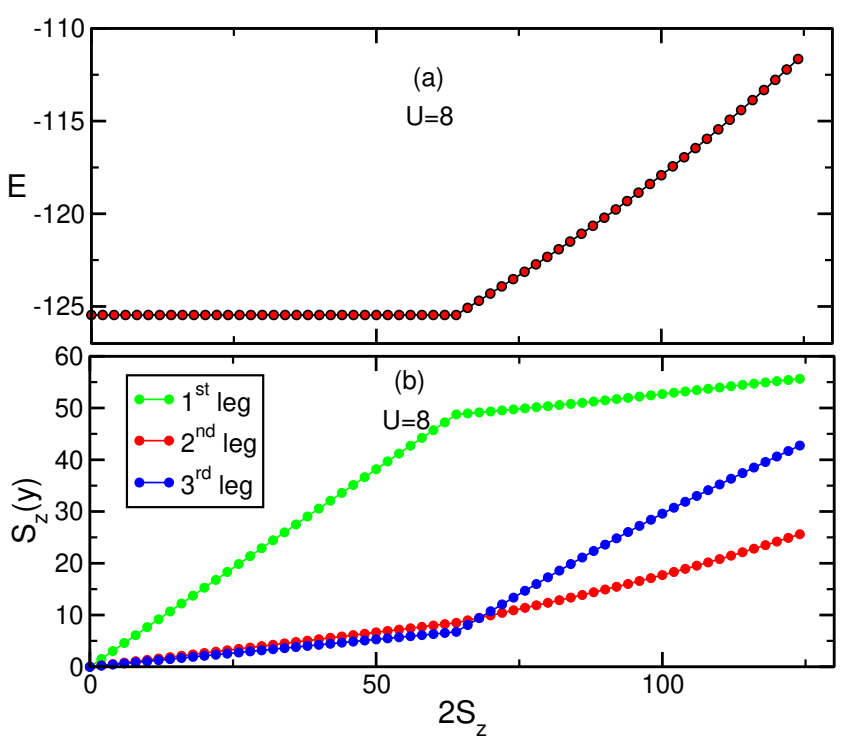

FIG. 5. (a) Energy of the lowest eigenstate and (b) its total spin density $S_{z}(y)$ on each leg of the 3-2 ladder geometry as a function of the $z$-projection of the eigenstate total spin $S_{z}$ for $L_{x}=128$, half filling and $U=8$. Note that the energy $E$ is an even function of $S_{z}$ while the densities are odd functions of $S_{z}$.

of the total spin $S_{z}=\left(N_{\uparrow}-N_{\downarrow}\right) / 2$. Results for the corresponding eigenenergies are shown in Fig. 5(a) for $U=8$ and $L_{x}=128$. The ground state is degenerate for all $\left|S_{z}\right| \leq S=32$ and thus ferromagnetic, in agreement with Lieb's prediction. For $\left|S_{z}\right|>32$ the eigenstates are excited states. As $S$ increases proportionally to the ladder length $L_{x}$, the ground state is macroscopically degenerate in the thermodynamic limit.

The DMRG method can be used to compute the charge and spin density profiles, defined by

$$
\begin{gathered}
N(x, y)=\left\langle n_{x, y, \uparrow}+n_{x, y, \downarrow}\right\rangle \\
S_{z}(x, y)=\left\langle n_{x, y, \uparrow}-n_{x, y, \downarrow}\right\rangle
\end{gathered}
$$

where $\langle\ldots\rangle$ denotes the expectation value in the lowest eigenstate for a given number $N_{\sigma}$ of electrons of each spin in the system. Due to the particle-hole symmetry of the Hamiltonian (5), the charge density is distributed homogeneously $N(x, y)=1$ at half-filling, despite the nominal inequivalence of different sites. This is even the case for arbitrary, unequal values of the nearest-neighbor hopping terms $t_{i}$.

The behavior of the total spin density for each leg

$$
S_{z}(y)=\sum_{x} S_{z}(x, y)
$$

is, however, non-trivial. $S_{z}(y)$ is depicted in Fig. 5(b) as a function of the $z$-projection of the total spin, for $L_{x}=128$ and $U=8$. We see that the spin density on each leg increases linearly for the values $\left|S_{z}\right| \leq$ 


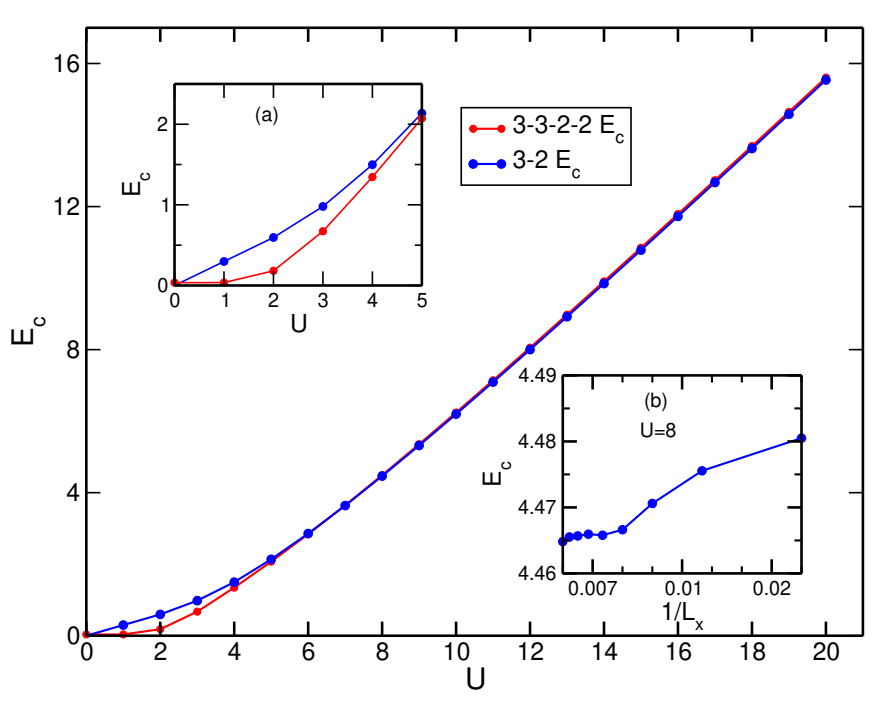

FIG. 6. Charge gap for the 3-2 and 3-3-2-2 ladders as a function of the interaction strength $U$ for $L_{x}=128$ at halffilling. The inset (a) shows the small coupling region on a smaller scale. The inset (b) shows the charge gap $E_{c}$ of the 3-2 ladder geometry at half-filling as a function of the inverse ladder length $1 / L_{x}$ for $U=8$.

$L_{x} / 4$, corresponding to the degenerate ground state in Fig. 5(a). For higher $S_{z}$, corresponding to excited states, the increase continues linearly but with a different slope. Most of the spin density is concentrated on the first leg for $0<\left|S_{z}\right| \leq L_{x} / 4$. Thus the ferromagnetic state is due to the unpaired electrons localized on this first leg. We remark that the macroscopic degeneracy in the thermodynamic limit is a consequence of Lieb's theorem in which our numerical results agree with it.

Additional information is provided by the gaps to the various excitations. The spin gap corresponds to the lowest excitation energy from the ground state with $S_{z}=0$ to an excited state in which the up and down fermion numbers differ by one $\left(S_{z}=1\right)$. That is,

$$
E_{s}=E\left(N_{\sigma}+1, N_{\sigma}-1\right)-E\left(N_{\sigma}, N_{\sigma}\right)
$$

where $E\left(N_{\uparrow}, N_{\downarrow}\right)$ is the ground-state energy for a Hubbard lattice with $N_{\sigma}$ electrons of each spin $\sigma$. Experimentally, the spin gap $E_{s}$ can be determined from the dynamical spin structure factor measured using inelastic neutron scattering [23].

The charge gap is the lowest excitation energy from the $N_{e}$-particle ground state to the $\left(N_{e} \pm 2\right)$-particle ground states with the same $S_{z}$. Its experimental value is deducible from, for example, the gap in the dynamical charge structure factor measured using electron-energyloss spectroscopy [24]. It is defined as [25]

$$
\begin{aligned}
E_{c} & =\frac{1}{2}\left[E\left(N_{\uparrow}+1, N_{\downarrow}+1\right)+E\left(N_{\uparrow}-1, N_{\downarrow}-1\right)\right] \\
& -E\left(N_{\uparrow}, N_{\downarrow}\right) .
\end{aligned}
$$

Finally, the single-particle gap is the lowest excitation energy $E_{p}$ seen in the single-particle spectral function, which can be measured in experiments such as Angle Resolved Photo-emission Spectroscopy (ARPES) [26]

$$
\begin{aligned}
E_{p} & =E\left(N_{\uparrow}+1, N_{\downarrow}\right)+E\left(N_{\uparrow}-1, N_{\downarrow}\right) \\
& -2 E\left(N_{\uparrow}, N_{\downarrow}\right) .
\end{aligned}
$$

Consequently, $E_{p}$ is the gap due to the excitation of a single-electron (with both charge and spin features) from the highest level below the Fermi level to the lowest level above the Fermi level.

The three gaps vanish in the half-filled noninteracting 3-2 ladder geometry. The spin gap remains small for a coupling $U>0$ at finite system size $L_{x}$ and extrapolates to zero within numerical accuracy. This is consistent with the degeneracy of the ferromagnetic ground state. An interaction $U>0$ generates a gap to the lowest charge excitations. The charge gap $E_{c}$, depicted in Fig. 6 for $L_{x}=128$, evolves monotonically with increasing $U$. $E_{c}$ extrapolates to finite values for $1 / L_{x} \rightarrow 0$ (see the inset (b) of Fig. 6) in agreement with Lieb's prediction [9]. The small finite size slope reflects the low velocity (almost zero) of the lowest excitations in this system. As for the homogeneous ladder, the charge gap is roughly linear in $U$ at strong coupling. Finally, the single-particle gap $E_{p}$ extrapolates to the same finite value as the charge gap $E_{c}$ for $1 / L_{x} \rightarrow 0$ with very small finite size effects. This behavior of excitation gaps is characteristic for the insulating ferromagnetic phase of the 3-2 geometry

Although at half-filling the particle-hole symmetry implies that the charge density is distributed homogeneously between the three legs, this symmetry is lost away from half filling. Using DMRG we calculate the change $\Delta N(x, y)$ in the charge density distribution $N(x, y)$ when two electrons are added to a half-filled ladder. Figure 7 shows the results for each leg of the half-filled 3-2 ladder at maximal $S_{z}$. The added charges are mainly on the third and second legs. One sees the presence of a double density peak that is typical for two independent particles in a box. This suggests that the two added electrons do not bind in this system in agreement with the vanishing of the pair binding energy in the thermodynamic limit $\left(E_{c}=E_{p}\right)$. Note that the asymmetric distribution $\Delta N(x, y)$ (i.e. added charge not centered at $x=L_{x} / 2=64$ ) is due to a poor DMRG convergence and reveals that the charge excitation band width is narrow as already suggested by the small finite-size corrections to the excitation gaps in Fig. 6, inset(b).

According to Shen et al. [10] a half-filled Hubbard model on a bipartite lattice with $N_{A} \neq N_{B}$ should exhibit a ferrimagnetic long-range order. More precisely, spins on the same sublattice should be ferromagnetically ordered while spin pairs on different sublattices should be antiferromagnetically ordered. This exact result applies to the 3-2 ladder geometry. Thus we should be able to observe this one-dimensional long-range order although it breaks the continuous $\mathrm{SU}(2)$ symmetry of the spin sector in the Hubbard model. 


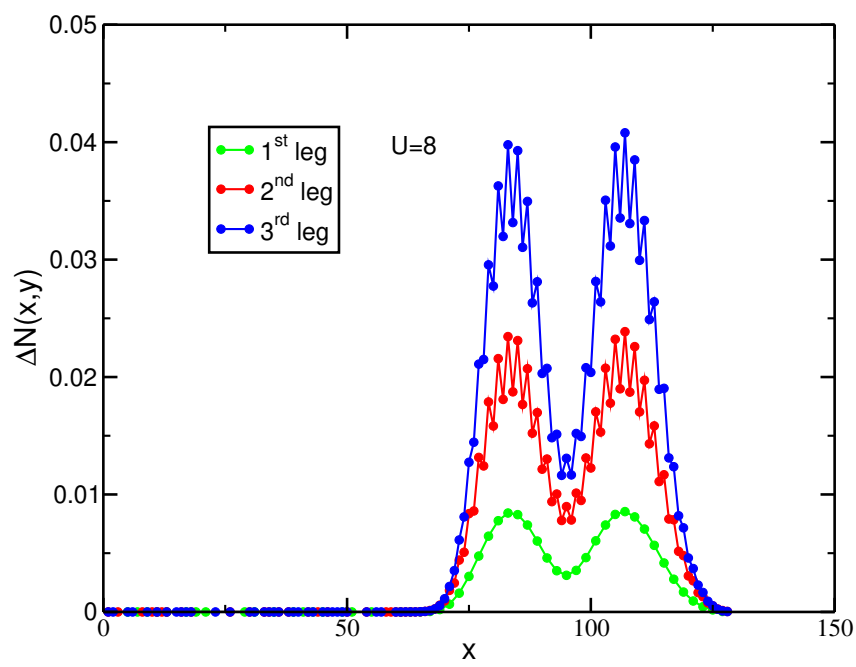

FIG. 7. Change $\Delta N(x, y)$ in the charge density distribution $N(x, y)$ on the three legs $(y=1,2,3)$ when two electrons are added to a half-filled 3-2 ladder with $U=8, L_{x}=128$, and $S_{z}=32$.

For this purpose we now investigate the groundstate spin correlations. The transverse spin correlation function between a site $x_{0}, y=1$ and the other sites $x_{0}+x, y$ is defined by

$$
C_{y}^{+-}(x)=\left\langle c_{x_{0}, 1, \uparrow}^{\dagger} c_{x_{0}, 1, \downarrow} c_{x_{0}+x, y, \downarrow}^{\dagger} c_{x_{0}+x, y, \uparrow}\right\rangle .
$$

These correlations are calculated using the DMRG method with the reference site $x_{0}$ located in the center of the chain, i.e. $x_{0}=L_{x} / 2$, in order to minimize boundary effects.

According to Ref. [10] this correlation function should reveal the ferrimagnetic long-range order between the spins in the $S_{z}=0$ ground state. Figure 8 shows DMRG results for the first leg, $C_{1}^{+-}(x)$, for a halffilled 3-2 ladder with $S_{z}=0$ and several values of the interaction $U$. The correlations decay very slowly with increasing distance $x$ as shown by the log-log scale. A fit to a power-law function yields very small exponents $|p| \lesssim 0.07$ as seen in the inset of Fig. 8. Antiferromagnetic correlations decrease as $1 / x$ in the antiferromagnetic isotropic Heisenberg chain. Fig. 8 indicates our 3-2 ladder system corresponds to the ferromagnetic isotropic Heisenberg chain. In both these models the ground state is macroscopically degenerate and has true long-range ferromagnetic order. If you approach the isotropic point of the Heisenberg chain from its Luttinger liquid phase, ferromagnetic correlations decay as $x^{-p}$ with an exponent $p$ that vanishes at the isotropic point [12]. While the homogeneous 3-leg ladder is in the universality class of the antiferromagnetic Heisenberg chain, the novel 3-2 ladder considered here seems to be in the class of the ferromagnetic chain.

The correlation functions for the second and third legs, $C_{y}^{+-}(x)$ for $y=2,3$, reveal long-range antiferromagnetic

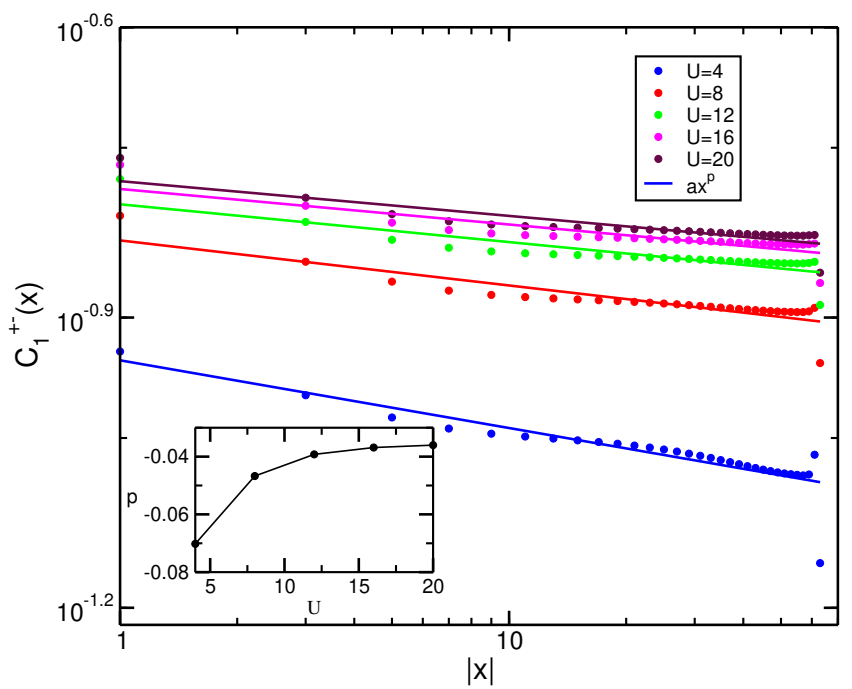

FIG. 8. Transverse spin correlation function $C_{1}^{+-}(x)$ in the $S_{z}=0$ ground state of the the 3-2 alternating ladder. Dots show the DMRG results calculated at half-filling for $L_{x}=$ 128 and various values of $U$. The straight lines represent the fitting function $a x^{p}$. The inset shows the exponent $p$ as a function of $U$.

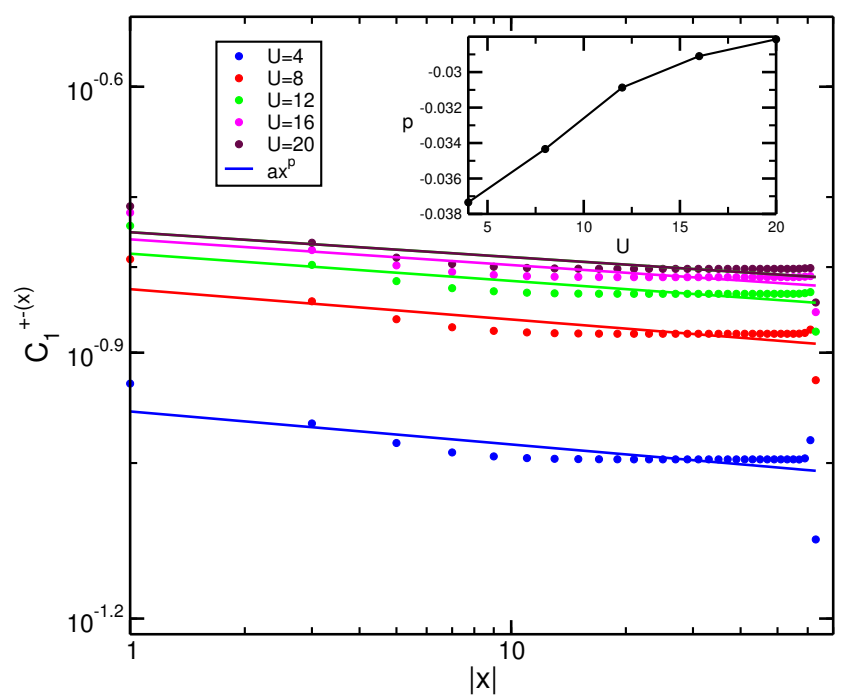

FIG. 9. Transverse spin correlation function $C_{1}^{+-}(x)$ in the $S_{z}=S=32$ ground state of the the 3-2 alternating ladder. Dots show DMRG results at half-filling for $L_{x}=128$ and various values of $U$. The straight lines represent the fitting function $a x^{p}$. The inset shows the exponent $p$ as a function of $U$.

correlations (not shown). Thus our results for the transverse correlation functions $C_{y}^{+-}(x)$ in the $S_{z}=0$ ground state agree with the exact results [10].

To the best of our knowledge there is no concrete prediction for the correlation functions $C_{y}^{+-}(x)$ in the ground states with $S_{z} \neq 0$. However, the exact results of Ref. [10] imply that the $\mathrm{SU}(2)$ invariant correlation 


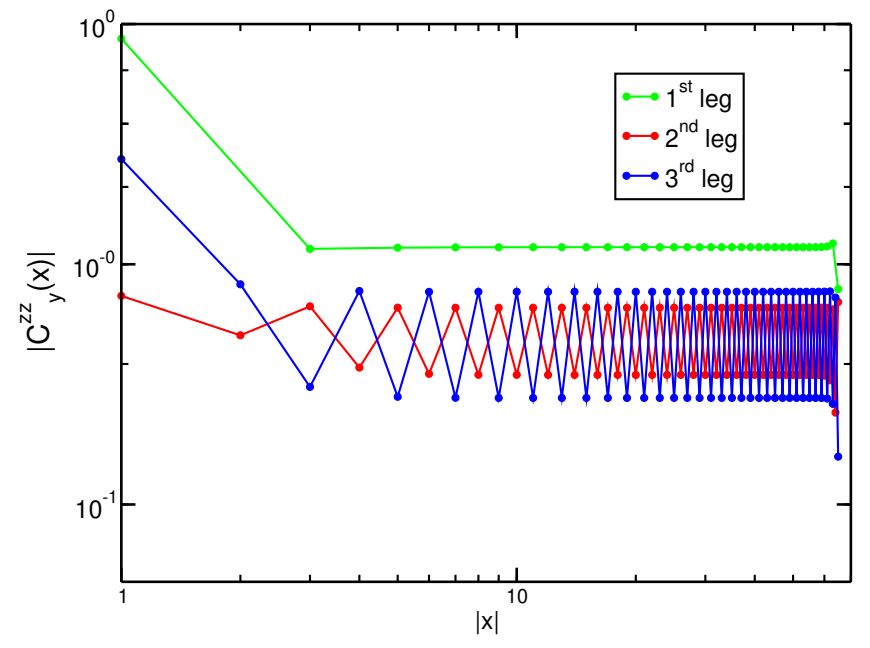

FIG. 10. Longitudinal spin correlation function $C_{y}^{z z}(x)$ in the $S_{z}=S=32$ ground state of the the 3-2 alternating ladder. Dots show DMRG results at half-filling for $L_{x}=128$ and $U=8$.

function

$$
C_{y}(x)=\left\langle\vec{S}_{x_{0}, y} \cdot \vec{S}_{x_{0}+x, y}\right\rangle
$$

must also exhibit long-range ferrimagnetic order for all ground states $-S \leq S_{z} \leq S$. Thus $C_{y}^{+-}(x)$, and the complementary longitudinal correlation function

$$
C_{y}^{z z}(x)=\left\langle\left(n_{x_{0}, 1, \uparrow}-n_{x_{0}, 1, \downarrow}\right)\left(n_{x_{0}+x, y, \uparrow}-n_{x_{0}+x, y, \downarrow}\right)\right\rangle,
$$

must display long-range ferrimagnetic order.

Our DMRG results reveal that the spin correlation $C_{y}^{+-}(x)$ decay very slowly for ground states with spin $S_{z} \neq 0$. Figure 9 shows the correlation function $C_{1}^{+-}(x)$ on the first leg for the ground state with maximal spin $S_{z}=32$. The exponents of power-law fits, $|p| \lesssim 0.04$, are even smaller than for the $S_{z}=0$ ground state. Again, this is compatible with long-range ferromagnetic order in the thermodynamic limit.

Turning to the longitudinal correlation function $C_{y}^{z z}(x)$, we find that it decays rapidly for the $S_{z}=$ 0 ground state. For the ground states with $S_{z} \neq$ 0, however, $C_{y}^{z z}(x)$ can also reveal the long-range ferrimagnetic order. Figure 10 plots $C_{y}^{z z}(x)$ for all legs $y=1,2,3$ at fixed $U$ for the ground state with the maximal spin $S_{z}=S$. Figure 11 shows the behavior of these correlations on the first leg only, varying $U$. Clearly, the ferromagnetic correlations do not decay with distance. A power-law fit yields exponents $|p| \lesssim 0.005$, as seen in the inset of this figure.

The ferrimagnetic long-range order is not visible in the spin density (Eq. 7) of the $S_{z}=0$ ground state because the spin flip symmetry imposes $S_{z}(x, y)=0$. However, the existence of this ordering can be seen in the spin density of the other ground states, in particular for the

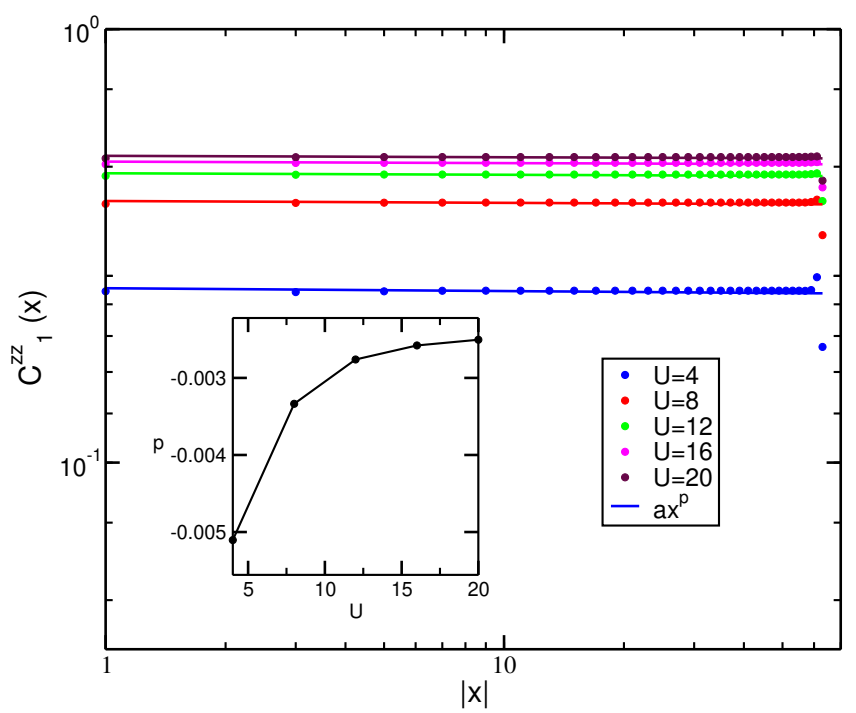

FIG. 11. Longitudinal spin correlation function $C_{1}^{z z}(x)$ in the $S_{z}=S=32$ ground state of the the 3-2 alternating ladder. Dots show DMRG results at half-filling for $L_{x}=128$ and various values of $U$. The straight lines represent the fitting function $a x^{p}$. The inset shows the exponent $p$ as a function of $U$.

maximal spin $S_{z}=S$. The sign of these spin densities alternates between nearest-neighbor sites as sketched in Fig. 12.

The ferromagnetic order parameter is the magnetization pro site $\left|N_{B}-N_{A}\right| /\left(N_{A}+N_{B}\right)$, which is independent of $U>0$. It is difficult to compute an order parameter for the antiferromagnetic ordering because the ground states are inhomogeneous in real space and break the spin rotation symmetry. In principle, one could compute the square root of the staggered average of the correlation function (13) over all sites for long ladder lengths $L_{x}$ but the computational cost would be excessive with our DMRG program.

In summary, our results for the 3-2 ladder geometry agree with the exact results in Refs. [9] and [10]. The ground state is gapped for charge excitations and ferromagnetic with a total spin $S \neq\left|N_{A}-N_{B}\right| / 2$. Moreover, the electron spins are ferrimagnetically ordered. Our results also quantify the precise behavior of the correlation functions.

\section{B. 3-3-2-2 alternating rung geometry}

We now turn to the Hubbard model on the 3-3-2-2 ladder geometry. This structure allows for unequal rung lengths, but unlike the $3-2$ case, this system represents a bipartite lattice with $N_{A}=N_{B}$. We have verified using DMRG that the ground state for $U>0$ at half filling has spin $S=0$ and is not degenerate.

The charge gap $E_{c}$ increases with $U$ and is very close to the charge gap of the 3-2 ladder geometry for $U \geq 5 t$ 
as seen in Fig. 6 for a finite ladder length. For weak coupling the charge gap of the 3-3-2-2 ladder is clearly smaller than in the $3-2$ ladder. For $U \geq 4 t$ our DMRG data indicate that the charge gap of the 3-3-2-2 ladder remains finite in the thermodynamic limit. For smaller $U$ we cannot determine whether the charge gap vanishes or is only very small in the thermodynamic limit.

In contrast to the 3-2 system, spin excitations are gapped in the 3-3-2-2 ladder. As shown in Fig. 13 for a ladder of finite length $L_{x}=128$, the spin gap $E_{s}$ increases with $U \geq 2$, reaches a maximum around $U=8$ and then decreases $\sim t^{2} / U$ for strong coupling. This behavior is similar to the behavior found in homogeneous two-leg ladders [19]. DMRG numerical errors and finitesize effects are not negligible for weak coupling $U<2$ and are responsible for the nonmonotonic behavior of the spin gap. (This is the case for many numerical methods including Quantum Monte Carlo. Larger $U$ breaks degeneracies and eliminates finite size 'shell effects' which are present at $U=0$.) For $U \geq 4 t$ we clearly see in Fig. 14(a) that the spin gap converges to a finite value in the thermodynamic limit, in contrast to the noninteracting system.

In Fig. 15 we show the total spin density on each leg of the 3-3-2-2 ladder with $U=8$ and $L_{x}=128$ as a function of the total spin $S_{z}$. The unpaired electron spins are distributed over the three legs but the first leg density is significantly lower than for the second and third legs. Thus in the presence of an external magnetic field, the unpaired electron spins are mostly localized on the second and third leg in the 3-3-2-2 system, in contrast to the magnetization of the first leg in the $3-2$ system, see Fig. 5(b).

The behavior of the single-particle gap $E_{p}$ is very similar to that of the charge gap $E_{c}$. In contrast to the case of the 3-2 ladder, however, we observe a difference between both gaps in the 3-3-2-2 ladder. The pair binding energy [27] is defined as

$$
E_{p b}=E_{p}-E_{c}
$$

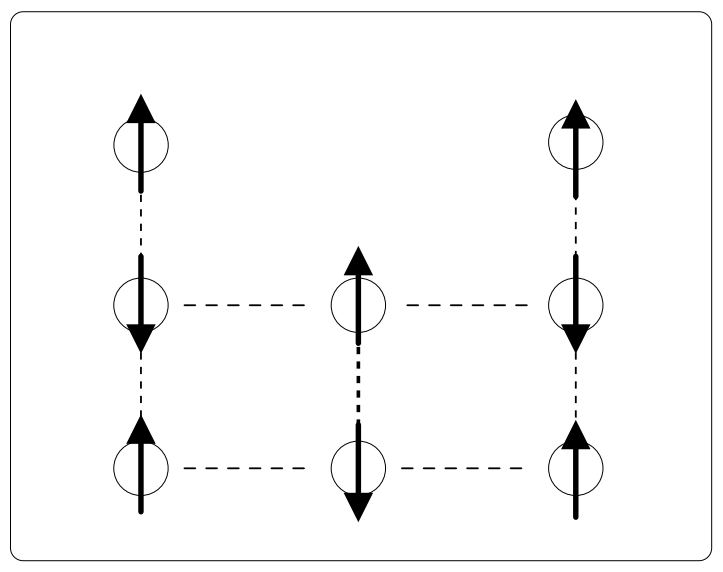

FIG. 12. Schematic illustration of the ferrimagnetic spin ordering in the 3-2 alternating ladder.

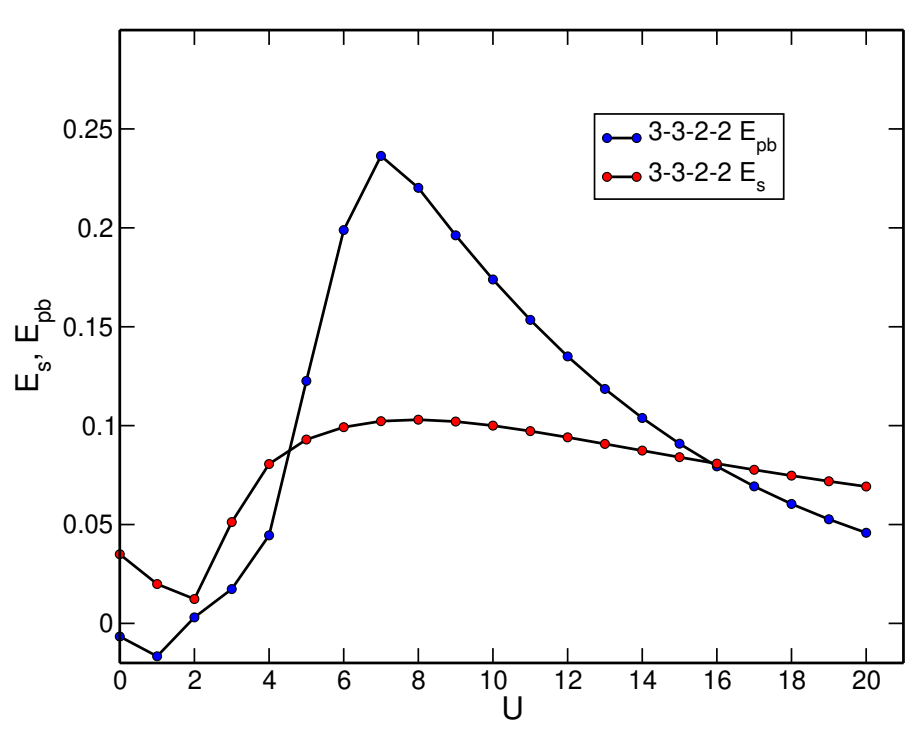

FIG. 13. Spin gap $E_{s}$ and pair-binding $E_{p b}$ energies of the half-filled 3-3-2-2 ladder with $L_{x}=128$ as a function of the coupling strength $U$.

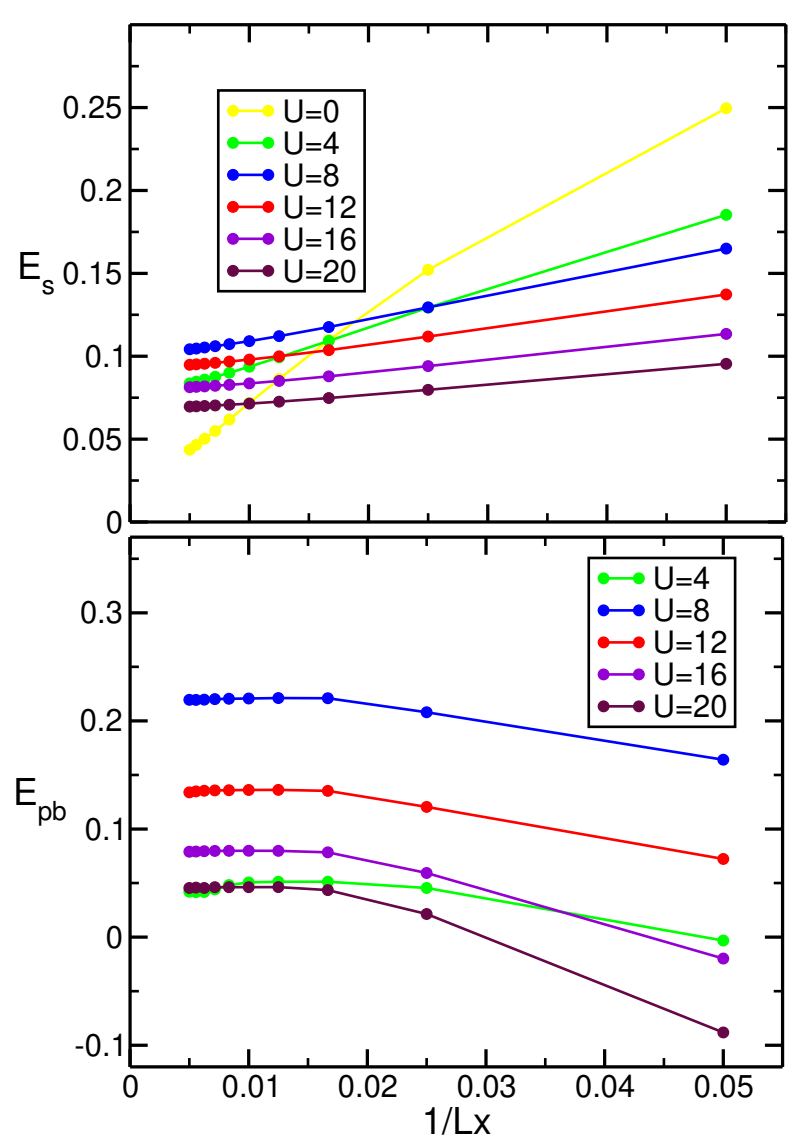

FIG. 14. (a) Spin gap of the half-filled 3-3-2-2 ladder as a function of the inverse ladder length $1 / L_{x}$ for different values of $U$. (b) Pair-binding energy $E_{p b}$ of the half-filled 3-3-2-2 ladder as a function of $1 / L_{x}$ for several values of $U$. 


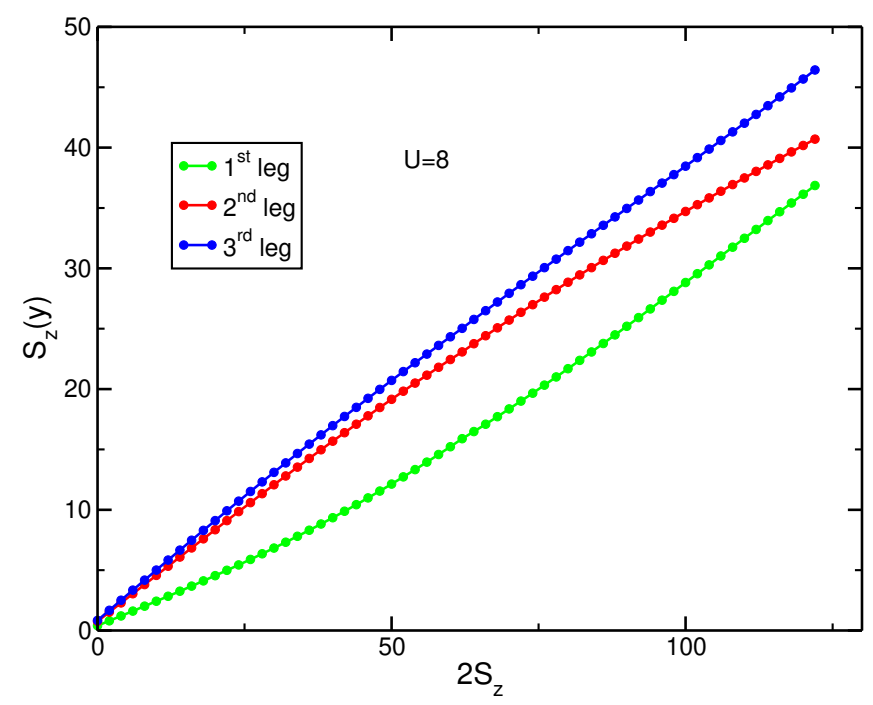

FIG. 15. Total spin density $S_{z}(y)$ on each leg of the half-filled 3-3-2-2 ladder with $U=8$ and $L_{x}=128$ as a function of the $z$-projection of the total spin.

Finite positive values of $E_{p b}$ indicate that it is energetically preferable for electrons or holes injected into the half-filled band to form pairs [28]. It has been shown for homogeneous 2-leg Hubbard ladders that the pair binding energy can be positive for some parameter ranges $[19,29,30]$. The finite-size scaling shown in Fig. 14(b) confirms that the pair-binding energy is positive and remains finite in the thermodynamic limit of the 3-3-2-2 ladder at least in the regime $U>4 t$. As seen in Fig. 13, the pair binding energy seems to reach its maximum $E_{p b} \approx 0.22 t$ for $U \approx 8$ where the spin gap $E_{s}$ is also the largest. Again this behavior is similar to the observation made for homogeneous symmetric [19] and anti-symmetric [31] two-leg Hubbard ladders. One can conclude that the pair binding energy is intimately related to the behavior of the spin gap energy [32].

As for the 3-2 system, the charge density $N(x, y)$ of the half-filled 3-3-2-2 ladder is uniformly distributed because of the particle-hole symmetry of the Hubbard Hamiltonian (5) on a bipartite lattice. Figure 16 shows the change $\Delta N(x, y)$ of this charge density distribution when two electrons are added to the half-filled system with $U=8$ and $L_{x}=128$. As was the case for the 3-2 system, the increase of the charge density in the first leg is smaller than the second and the third leg. The single peak resembles the distribution expected for a single particle in a box. This suggests that the two electrons build a pair (mostly localized on the second and third leg) in agreement with the observation of a finite binding energy. The asymmetric distribution $\Delta N(x, y)$ is due to a poor DMRG convergence as already discussed in the previous section.

Spin correlations decrease exponentially with distance in the half-filled 3-3-2-2 ladder, as expected for a spin gapped system. These correlations reveal short-

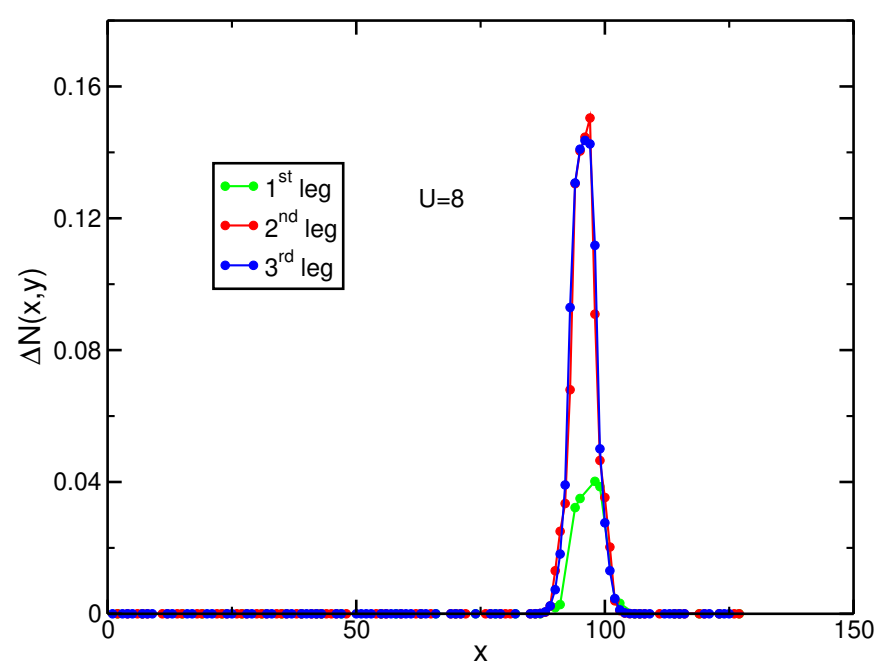

FIG. 16. Change $\Delta N(x, y)$ in the charge density distribution $N(x, y)$ on the three legs $(y=1,2,3)$ when two electrons are added to a half-filled 3-3-2-2 ladder with $U=8$ and $L_{x}=128$.

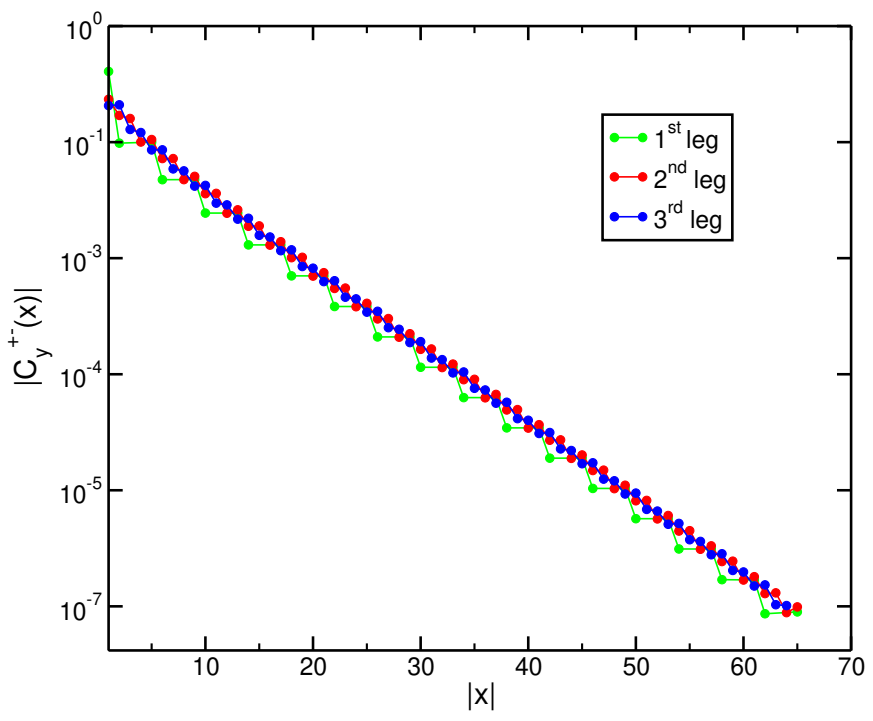

FIG. 17. Spin correlation function $C_{y}^{+-}(x)$ for the 3-3-2-2 geometry along each leg $y=1,2,3$ at half-filling with $U=8$ and $L_{x}=128$.

range antiferromagnetic order. Figure 17 illustrates the exponential decrease of the correlation function $C_{y}^{+-}(x)$ with the distance $x$ between two sites on each leg $y$ for $U=8$ and $L_{x}=128$. We see that the decay of spin correlations is similarly fast in all legs.

In Fig. 18, the exponential decay of spin correlations is plotted for different values of $U$. We derive a correlation length $\xi$ by fitting these data to an exponential function. The results are shown in the inset of Fig. 18 as a function of $U$. The correlation length decreases rapidly with increasing coupling $U$ but seems to saturate at a finite value for strong coupling. This can be explained by 
the fact that the correlation length is roughly given by the ratio between the band width and the gap of spin excitations. For weak $U$ we know that the band width is $\propto t$ while the spin gap is small, see Fig. 13, leading to a divergence of $\xi$ for $U \rightarrow 0$. For strong $U$, however, both the band width and the spin gap scale with the effective exchange coupling $J \propto t^{2} / U$.

In order to get information about the relative orientation of spins on nearest-neighbor sites, we calculate the spin bond order defined as,

$$
B\left(x, y, x^{\prime}, y^{\prime}\right)=\left\langle\left(n_{x, y, \uparrow}-n_{x, y, \downarrow}\right)\left(n_{x^{\prime}, y^{\prime}, \uparrow}-n_{x^{\prime}, y^{\prime}, \downarrow}\right)\right\rangle
$$

where $(x, y)$ and $\left(x^{\prime}, y^{\prime}\right)$ are nearest-neighbor sites.

Note that the isotropic spin bond order is equal to (16) up to a factor $3 / 4$ because the ground state of the 3-3-2-2 ladder geometry has spin $S=0$.

Figure 19 shows DMRG results for this spin bond order. We see that the spin bond is much stronger between the nearest-neighbor spins on the first leg than all other spin bonds. This suggests that each nearestneighbor pair on the first leg builds a strong singlet which is then weakly coupled to the rest of the system. The second and third legs build an effective two-leg ladder. This structure is illustrated in Fig. 19(b). This (partial) decoupling of the first leg from the other two legs explains the similarities between the 3-3-2-2 system and two-leg ladders that we observe, in particular the pair binding of two added electrons.

\section{CONCLUSION}

The alternation between gapped and ungapped spectra in uniform ladder systems has been of profound

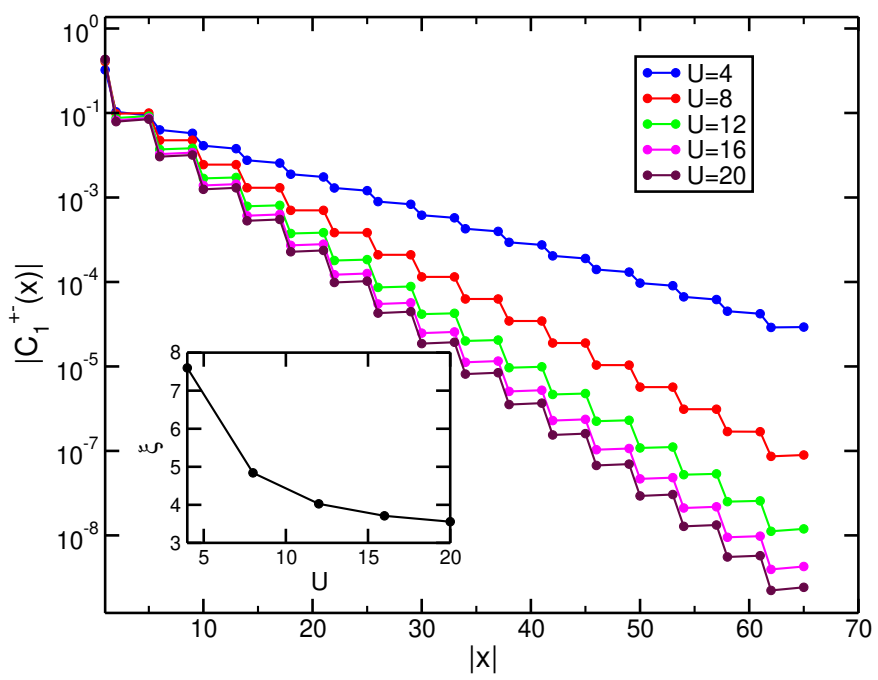

FIG. 18. Spin correlation function $C_{1}^{+-}(x)$ in the first leg for the 3-3-2-2 geometry at half-filling for various values of $U$. The inset shows the derived correlation length $\xi$ as a function of $U$.
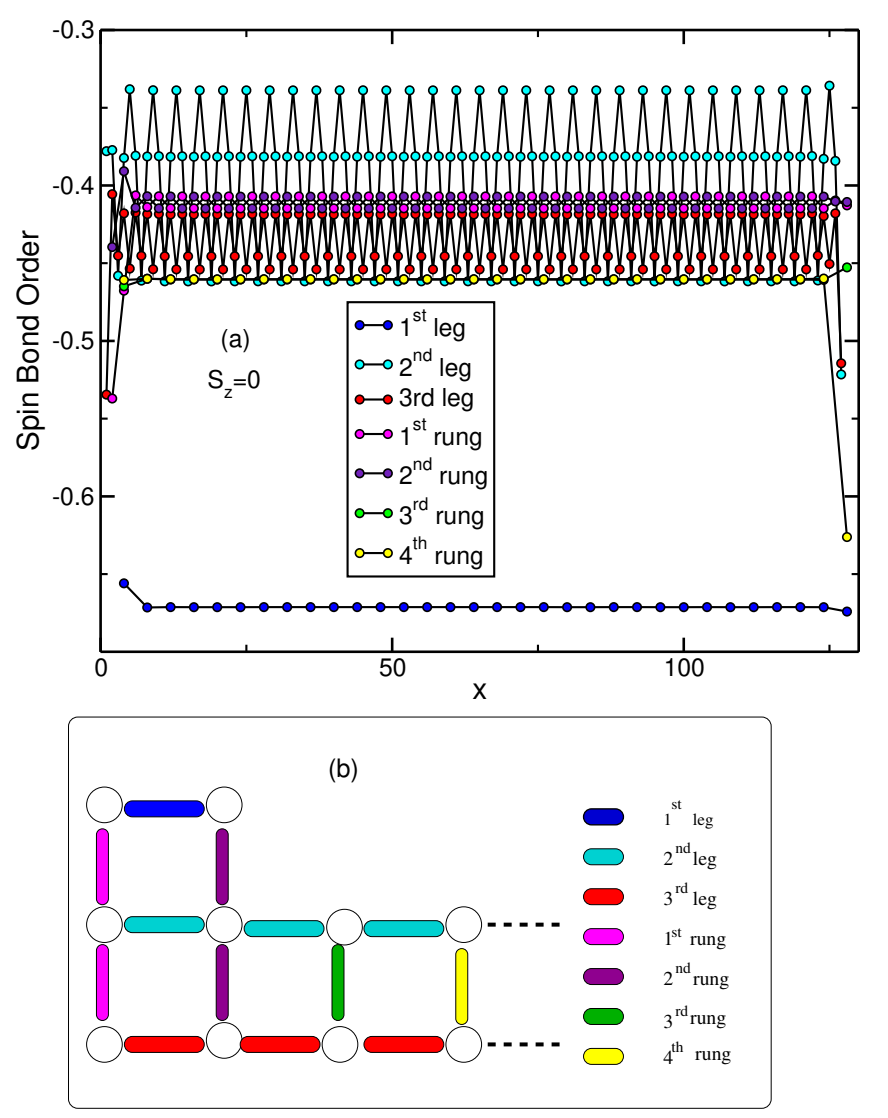

FIG. 19. (a) Spin bond order between nearest-neighbor sites for the 3-3-2-2 ladder at half-filling with $U=8$ and $L_{x}=128$. (b) Schematic illustration of the 3-3-2-2 configuration.

importance in condensed matter physics [11, 33-39]. The focus of the present work was to explore spin and charge properties of novel Hubbard ladder systems whose geometry consists of alternating numbers of sites per rung and thus 'mix' the structures which are associated with these two situations. Analogs of such structures, chains with spins alternating between $S=1 / 2$ and $S=1$, have revealed a number of unexpected phenomena driven by a competition between half-integer and integer spin physics, including transitions between antiferromagnetic and ferromagnetic behavior as the temperature is varied [40-43]. Here we can investigate such physics for itinerant electrons rather than quantum spins, in geometries which combine features of odd and even rung systems. Our consideration of the Hubbard rather than the Heisenberg Hamiltonian also allows us to consider the effects of the unique zero energy bands associated with bipartite lattices with unequal number of sites per sublattice. Our findings are consistent with available analytical results for such geometries $[9,10]$.

Investigating two different geometries has allowed us to isolate the effects of unequal sublattice site numbers from that of a periodically varying number of sites per rung. We have found that the low-energy properties of the two three-leg ladder systems at half filling differ 
radically. The magnetic properties of an alternating ladder with unequal number of sites in each sublattice are similar to those predicted for the Hubbard model on a Lieb lattice. The ground state has a total spin $S$ proportional to the system size and the electron spins are ordered ferrimagnetically. Thus this is a rare example of long-range magnetic order in a one-dimensional quantum system with short-range interactions. Moreover, we have found that electron or hole pairs added to the half filled system do not seem to bind. In contrast, the properties of the alternating ladder with equal number of sites in each sublattice resemble the properties found in two-leg ladders because the spins on the depleted leg tends to build strong singlets. The ground state is paramagnetic and non-degenerate. Added electron and hole pairs tend to bind with a binding energy that seems to be set by the size of the spin gap.

Past investigations of uniform spin and fermion ladders already revealed profoundly different low energy magnetic properties, e.g. depending on the number of legs [12]. Attention subsequently turned to refinements including DMRG calculations that revealed transitions between gapped paramagnetic and ferrimagnetic phases in two-leg ladders with alternating spin-1/2 and spin-1 degrees of freedom [40, 44-46]. Our work shows that ladders with varying number of sites per rung exhibit a similar rich physics, including long-range magnetic order, while being amenable to well-established methods for one-dimensional quantum many-body systems.

It will be interesting to explore further aspects of the physics of uniform ladders in our alternating geometry, including both how pairing correlations decay [47, 48], and also the nature of spin correlations in the vicinity of magnetic impurities[49].

\section{ACKNOWLEDGMENTS}

The work of R.S. was supported by the grant DESC0014671 funded by the U.S. Department of Energy, Office of Science.

\section{Appendix A: Homogeneous 3-leg ladder}

Here we review some results for homogeneous noninteracting 3-leg ladders and detail the method that we also use to study the alternating ladder geometries in Sec. II. The structure of the homogeneous 3-leg ladder lattice is shown in Fig 20. It consists of three chains, with intra-chain hopping $t$ and inter-chain hopping $t^{\prime}$. We focus on the band structure and DOS of this system with $t=t^{\prime}=1$ for both noninteracting and interacting cases.

If we use periodic boundary conditions in the legdirection and the non-interacting Hamiltonian (1) is periodic with period $d$ in that direction, we can write

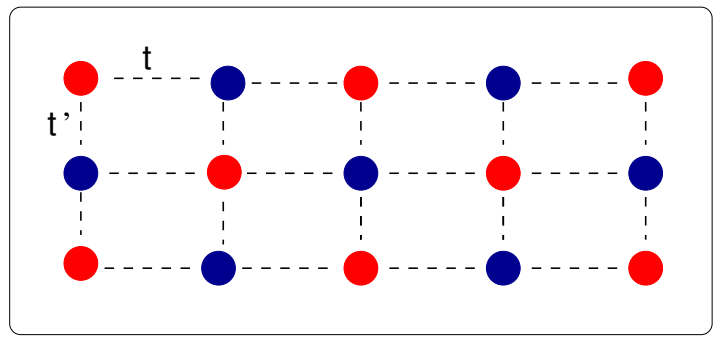

FIG. 20. Homogeneous 3-leg ladder with intra-leg hopping term $t$ and inter-leg hopping term $t^{\prime}$.

it as a sum

$$
H_{0}=\sum_{k} H_{k}
$$

of commuting many-body operators $H_{k}$. Each $H_{k}$ acts only on the single-particle Bloch states with the wave number

$$
k=\frac{2 \pi j d}{L_{x}}
$$

in the first Brillouin zone where the quantum number $j$ satisfies $-L_{x} /(2 d)<j \leq L_{x} /(2 d)$. $L_{x}$ is the number of rungs and $N_{c}=L_{x} / d$ is the number of unit cells or equivalently the number of wave numbers $k$. The Bloch states are given by the transformation

$$
d_{k, x, y, \sigma}^{\dagger}=\sqrt{\frac{d}{L_{x}}} \sum_{n=1}^{L_{x} / d} c_{x+n d, y, \sigma}^{\dagger} \exp (i k n) .
$$

where $x=1, \ldots, d$. For the homogeneous 3-leg ladder we have $d=1$. Each many-body Hamiltonian $H_{k}$ acts on as many single-particle states as there are sites in one unit cell. For the homogeneous 3-leg ladder this dimension is three. Thus we obtain the $3 \times 3$ matrix representation of $H_{k}$ for single-particle states

$$
H_{k}^{(1)}=\left(\begin{array}{ccc}
-2 t \cos (k) & -t^{\prime} & 0 \\
-t^{\prime} & -2 t \cos (k) & -t^{\prime} \\
0 & -t^{\prime} & -2 t \cos (k)
\end{array}\right) .
$$

In order to gain some insight into this system, we calculate the single-particle eigenenergies by diagonalizing this matrix, which leads to three bands with the dispersion relation

$$
E_{k, b}=-2 t \cos (k)+\varepsilon_{b}
$$

where $\varepsilon_{b}=0, \pm \sqrt{2} t^{\prime}$. The index $b(=1,2,3)$ numbers the bands. The band structure is shown in Fig. 21 (a). As we consider a homogeneous ladder with inequivalent legs, it is useful to calculate the leg-resolved DOS

$$
N(E, y)=\frac{1}{N_{c}} \sum_{k, b}\left|\psi_{k, b}(y)\right|^{2} \delta\left(E-E_{k, b}\right)
$$



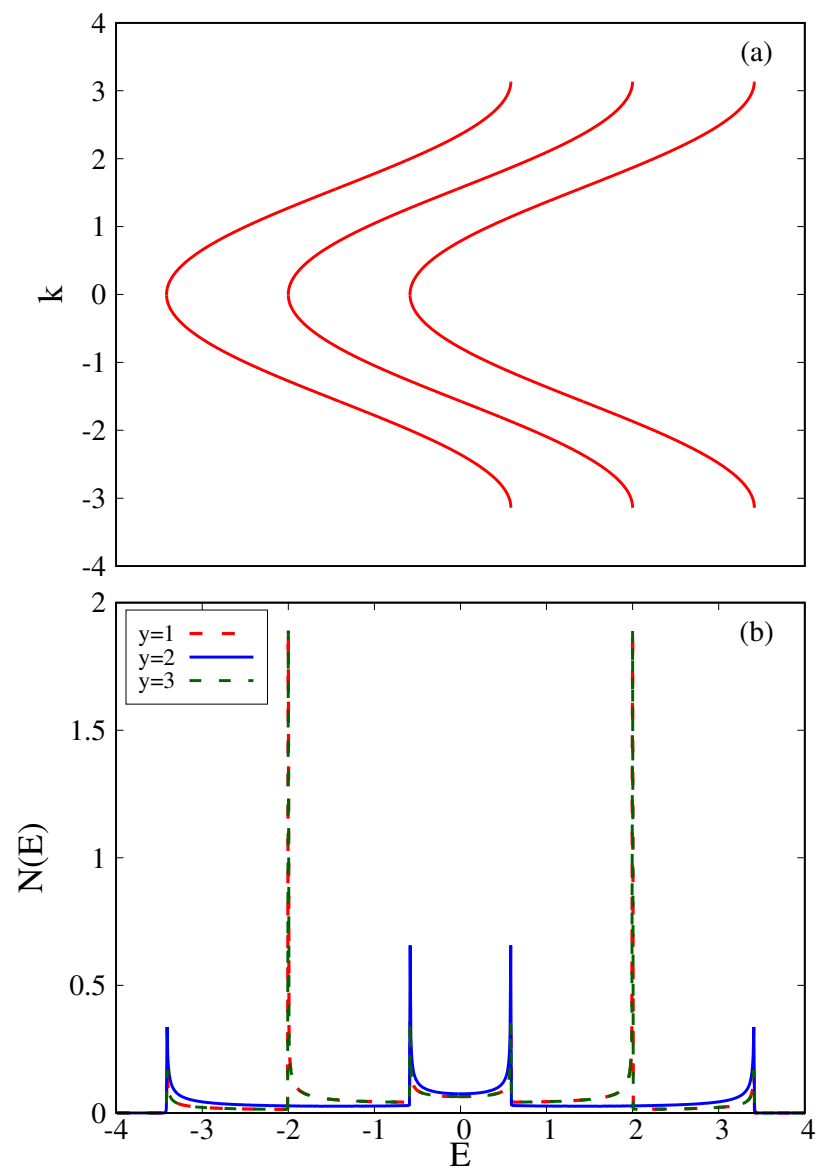

FIG. 21. (a) Band structure for the noninteracting homogeneous 3-leg ladder with $t=t^{\prime}=1$. (b) Corresponding density of states on each leg $y=1,2,3$. Note that $N(E, 1)=$ $N(E, 3)$. for $y=1,2,3$ rather than the total DOS (4). Here $\psi_{k, b}(y)$ represents the eigenvector of the matrix (A4) corresponding to the eigenenergy $E_{k, b}$ and $N_{c}=L_{x}$. The DOS for the homogeneous 3-leg ladder is plotted in Fig. 21(b). It shows six Van Hove singularity peaks for the first and third leg, with $N(E, 1)=$ $N(E, 3)$. The DOS on the second leg exhibits only four singularities because the band (A5) with $\varepsilon_{b}=0$ is antisymmetric under reflection in the $y$-direction and thus the corresponding single-particle eigenstates vanish on the middle leg, $\psi_{k, b}(x, y=2)=0$. For all finite values of $t$ and $t^{\prime}$ the system is metallic, i.e. there is at least one band crossing the Fermi level $E_{F}=0$ at half filling.

\section{Appendix B: Hamiltonian matrix for the noninteracting 3-3-2-2 ladder geometry}

The single-particle matrix representation $H_{k}^{(1)}$ of the Hamiltonians $H_{k}$ for the noninteracting 3-3-2-2 ladder geometry is the following $10 \times 10$ matrix with $\widetilde{t}_{j}=$ $t_{j} \exp (i k)$.

$$
H_{k}^{(1)}=\left(\begin{array}{cccccccccc}
0 & -t_{1} & 0 & 0 & 0 & -t_{5} & 0 & 0 & 0 & \widetilde{t}_{15} \\
-t_{1} & 0 & -t_{2} & 0 & -t_{4} & 0 & 0 & 0 & \widetilde{t}_{14} & 0 \\
0 & -t_{2} & 0 & -t_{3} & 0 & 0 & 0 & 0 & 0 & 0 \\
0 & 0 & -t_{3} & 0 & -t_{6} & 0 & 0 & 0 & 0 & 0 \\
0 & -t_{4} & 0 & -t_{6} & 0 & -t_{7} & 0 & -t_{8} & 0 & 0 \\
-t_{5} & 0 & 0 & 0 & -t_{7} & 0 & -t_{9} & 0 & 0 & 0 \\
0 & 0 & 0 & 0 & 0 & -t_{9} & 0 & -t_{10} & 0 & -t_{12} \\
0 & 0 & 0 & 0 & -t_{8} & 0 & -t_{10} & 0 & -t_{11} & 0 \\
0 & -\widetilde{t}_{14}^{*} & 0 & 0 & 0 & 0 & 0 & -t_{11} & 0 & -t_{13} \\
-\widetilde{t}_{15}^{*} & 0 & 0 & 0 & 0 & 0 & -t_{12} & 0 & -t_{13} & 0
\end{array}\right)
$$

1 R. Toskovic, R. van den Berg, A. Spinelli, I. S. Eliens, B. van den Toorn, B. Bryant, J. S. Caux, and A. F. Otte. Atomic spin-chain realization of a model for quantum criticality. Nature Physics, 12:656, 04 2016. URL https: //doi.org/10.1038/nphys3722.
${ }^{2}$ P. Gambardella, A. Dallmeyer, K. Maiti, M. C. Malagoli, W. Eberhardt, K. Kern, and C. Carbone. Ferromagnetism in one-dimensional monatomic metal chains. Nature, 416 (6878):301-304, 2002. doi:10.1038/416301a. URL https : //doi.org/10.1038/416301a. 
${ }^{3}$ Marlou R. Slot, Thomas S. Gardenier, Peter H. Jacobse, Guido C. P. van Miert, Sander N. Kempkes, Stephan J. M. Zevenhuizen, Cristiane Morais Smith, Daniel Vanmaekelbergh, and Ingmar Swart. Experimental realization and characterization of an electronic Lieb lattice. Nature Physics, 13:672, 04 2017. URL https: //doi.org/10.1038/nphys4105.

4 John N Randall, James H G Owen, Joseph Lake, Rahul Saini, Ehud Fuchs, Mohammad Mahdavi, S O Reza Moheimani, and Benjamin Carrion Schaefer. Highly parallel scanning tunneling microscope based hydrogen depassivation lithography. Journal of Vacuum Science E) Technology B, 36:6-10, 2018. ISSN 21662754. doi: 10.1116/1.5047939. URL http://avs.scitation.org/ toc/jvb/36/6.

5 Wiley P. Kirk, John N. Randall, and James H. G. Owen, editors. 2D Quantum Metamaterials, April 2019. World Scientific. doi:10.1142/11438. Proceedings of the 2018 NIST Workshop.

${ }^{6}$ Immanuel Bloch. Ultracold quantum gases in optical lattices. Nature Physics, 1:23, 2005. doi:10.1038/nphys138.

7 Immanuel Bloch, Jean Dalibard, and Wilhelm Zwerger. Many-body physics with ultracold gases. Rev. Mod. Phys., 80:885-964, July 2008. doi:10.1103/RevModPhys.80.885.

8 Markus Greiner and Simon Fölling. Optical lattices. Nature, 453:736, 2008. doi:10.1038/453736a.

9 Ellio Lieb. Two theorems on the Hubbard model. Phys. Rev. Lett., 62(10):1201, 1989.

10 Shun-Qing Shen, Zhao-Ming Qiu, and Guang-Shan Tian. Ferrimagnetic long-range order of the Hubbard model. Phys. Rev. Lett., 72:1280-1282, Feb 1994. doi: 10.1103/PhysRevLett.72.1280. URL https://link.aps . org/doi/10.1103/PhysRevLett.72.1280.

11 Elbio Dagotto and TM Rice. Surprises on the way from one-to two-dimensional quantum magnets: the ladder materials. Science, 271(5249):618-623, 1996.

12 Thierry Giamarchi. Quantum physics in one dimension, volume 121. Oxford university press, 2004.

13 D Belitz and TR Kirkpatrick. The Anderson-Mott transition. Reviews of Modern Physics, 66(2):261, 1994.

14 Kenro Kawano and Minoru Takahashi. Three-leg antiferromagnetic Heisenberg ladder with frustrated boundary condition; ground state properties. Journal of the Physical Society of Japan, 66(12):4001-4008, 1997.

15 Jie Xu, Chia-Chen Chang, Eric J Walter, and Shiwei Zhang. Spin-and charge-density waves in the HartreeFock ground state of the two-dimensional Hubbard model. Journal of Physics: Condensed Matter, 23(50):505601, 2011.

16 Fabian HL Essler, Holger Frahm, Frank Göhmann, Andreas Klümper, and Vladimir E Korepin. The onedimensional Hubbard model. Cambridge University Press, 2005.

17 Barry Friedman. A density matrix renormalization group approach to interacting quantum systems on Cayley trees. Journal of Physics: Condensed Matter, 9(42):9021, 1997.

18 T. Xiang. Density-matrix renormalization-group method in momentum space. Phys. Rev. B, 53:R10445-R10448, Apr 1996. doi:10.1103/PhysRevB.53.R10445. URL https: //link.aps.org/doi/10.1103/PhysRevB.53.R10445.

19 E Jeckelmann, DJ Scalapino, and SR White. Comparison of different ladder models. Phys. Rev. B, 58(14):9492, 1998.

20 Steven R White. Density-matrix algorithms for quantum renormalization groups. Phys. Rev. B, 48(14):10345, 1993.
21 Ulrich Schollwöck. The density-matrix renormalization group. Reviews of Modern Physics, 77(1):259, 2005.

22 J Bonča, JE Gubernatis, M Guerrero, Eric Jeckelmann, and Steven R White. Stripes in a three-chain Hubbard ladder: A comparison of density-matrix renormalization group and constrained-path Monte Carlo results. Phys. Rev. B, 61(5):3251, 2000.

23 Albert Furrer, Joël Mesot, and Thierry Strässle. Neutron scattering in condensed matter physics. World Scientific Publishing Company, 2009.

24 Ray F Egerton. Electron energy-loss spectroscopy in the electron microscope. Springer Science \& Business Media, 2011.

25 RM Noack, SR White, and DJ Scalapino. The doped two-chain Hubbard model. Europhysics Letters, 30(3):163, 1995.

26 Wentao Zhang. Photoemission Spectroscopy on High Temperature Superconductor: A Study of Bi2Sr2CaCu2O8 by Laser-Based Angle-Resolved Photoemission. Springer Science \& Business Media, 2012.

27 Sudip Chakravarty, Martin P Gelfand, and Steven Kivelson. Electronic correlation effects and superconductivity in doped fullerenes. Science, 254 (5034):970-974, 1991.

${ }^{28}$ Fei Lin, Erik S Sørensen, Catherine Kallin, and A John Berlinsky. Strong correlation effects in the fullerene $c_{2} 0$ studied using a one-band Hubbard model. Phys. Rev. B, 76(3):033414, 2007.

29 SN Coppersmith and C Yu Clare. Phase diagram of the Hubbard model: A variational wave-function approach. Phys. Rev. B, 39(16):11464, 1989.

30 S Chakravarty, L Chayes, and S.A. Kivelson. Absence of pair binding in the Hubbard model. Letters in Mathematical Physics, 23(4):265-270, 1991.

31 Anas Abdelwahab, Eric Jeckelmann, and Martin Hohenadler. Ground-state and spectral properties of an asymmetric Hubbard ladder. Phys. Rev. B, 91:155119, Apr 2015. doi:10.1103/PhysRevB.91.155119. URL https: //link.aps.org/doi/10.1103/PhysRevB.91.155119.

32 Karl-Heinz Bennemann and John B Ketterson. The Physics of Superconductors: Vol II: Superconductivity in Nanostructures, High-Tc and Novel Superconductors, Organic Superconductors, volume 2. Springer Science \& Business Media, 2011.

33 Yoshitami Ajiro, Tsuneaki Goto, Hikomitsu Kikuchi, Toshiro Sakakibara, and Toshiya Inami. Highfield magnetization of a quasi-one-dimensional $\mathrm{s}=1$ antiferromagnet $\mathrm{ni}\left(\mathrm{c}_{2} \mathrm{~h}_{8} \mathrm{n}_{2}\right)_{2} \mathrm{no}_{2}\left(\mathrm{clo}_{4}\right.$ : Observation of the haldane gap. Phys. Rev. Lett., 63:1424-1427, Sep 1989. doi: 10.1103/PhysRevLett.63.1424. URL https://link.aps . org/doi/10.1103/PhysRevLett.63.1424.

34 M. Azuma, Z. Hiroi, M. Takano, K. Ishida, and Y. Kitaoka. Observation of a spin gap in $\mathrm{Srcu}_{2} \mathrm{O}_{3}$ comprising spin-1/2 quasi-1d two-leg ladders. Phys. Rev. Lett., 73:3463-3466, Dec 1994. doi:10.1103/PhysRevLett.73.3463. URL https: //link.aps.org/doi/10.1103/PhysRevLett.73.3463.

35 L. P. Regnault, I. Zaliznyak, J. P. Renard, and C. Vettier. Inelastic-neutron-scattering study of the spin dynamics in the Haldane-gap system $\mathrm{Ni}\left(\mathrm{C}_{2} \mathrm{H}_{8} \mathrm{~N}_{2}\right)_{2} \mathrm{NO}_{2} \mathrm{ClO}_{4}$. Phys. Rev. B, 50:9174-9187, Oct 1994. doi:10.1103/PhysRevB.50.9174. URL https://link.aps.org/doi/10.1103/PhysRevB.50.9174.

36 SR White, RM Noack, and DJ Scalapino. Resonating valence bond theory of coupled Heisenberg chains. Phys. 
Rev. Lett., 73(6):886, 1994.

37 G. E. Granroth, M. W. Meisel, M. Chaparala, Th. Jolicœur, B. H. Ward, and D. R. Talham. Experimental evidence of a haldane gap in an $S=2$ quasi-linearchain antiferromagnet. Phys. Rev. Lett., 77:1616-1619, Aug 1996. doi:10.1103/PhysRevLett.77.1616. URL https: //link.aps.org/doi/10.1103/PhysRevLett.77.1616.

38 M. Takigawa, T. Asano, Y. Ajiro, M. Mekata, and Y. J. Uemura. Dynamics in the $S=1$ OneDimensional Antiferromagnet $\mathrm{AgVP}_{2} \mathrm{~S}_{6}$ via ${ }^{31} \mathrm{P}$ and ${ }^{51} \mathrm{~V}$ NMR. Phys. Rev. Lett., 76:2173-2176, Mar 1996. doi: 10.1103/PhysRevLett.76.2173. URL https://link.aps. org/doi/10.1103/PhysRevLett.76.2173.

39 M. Greven, R. J. Birgeneau, and U. J. Wiese. Monte carlo study of correlations in quantum spin ladders. Phys. Rev. Lett., 77:1865-1868, Aug 1996. doi: 10.1103/PhysRevLett.77.1865. URL https://link.aps . org/doi/10.1103/PhysRevLett.77.1865.

40 Swapan K. Pati, S. Ramasesha, and Diptiman Sen. Lowlying excited states and low-temperature properties of an alternating spin-1-spin-1/2 chain: A density-matrix renormalization-group study. Phys. Rev. B, 55:8894-8904, Apr 1997. doi:10.1103/PhysRevB.55.8894. URL https: //link.aps.org/doi/10.1103/PhysRevB.55.8894.

41 Shoji Yamamoto, S. Brehmer, and H.-J. Mikeska. Elementary excitations of Heisenberg ferrimagnetic spin chains. Phys. Rev. B, 57:13610-13616, Jun 1998. doi: 10.1103/PhysRevB.57.13610. URL https://link.aps. org/doi/10.1103/PhysRevB.57.13610.

42 Shoji Yamamoto and Takahiro Fukui. Thermodynamic properties of heisenberg ferrimagnetic spin chains: Ferromagnetic-antiferromagnetic crossover. Phys. Rev. B, 57:R14008-R14011, Jun $1998 . \quad$ doi:
10.1103/PhysRevB.57.R14008. URL https: //link.aps.org/doi/10.1103/PhysRevB.57.R14008.

43 A. Langari and M. A. Martín-Delgado. Alternating-spin ladders in a magnetic field: Formation of magnetization plateaux. Phys. Rev. B, 62:11725-11730, Nov 2000. doi: 10.1103/PhysRevB.62.11725. URL https://link.aps. org/doi/10.1103/PhysRevB.62.11725.

44 N. B. Ivanov. Spin-wave series for quantum onedimensional ferrimagnets. Phys. Rev. B, 57:R14024R14027, Jun 1998. doi:10.1103/PhysRevB.57.R14024. URL https://link.aps.org/doi/10.1103/PhysRevB. 57.R14024.

45 Takahiro Fukui and Norio Kawakami. Alternating-spin ladders. Phys. Rev. B, 57:398-402, Jan 1998. doi: 10.1103/PhysRevB.57.398. URL https://link.aps.org/ doi/10.1103/PhysRevB.57.398.

46 V. Ravi Chandra, N. B. Ivanov, and J. Richter. Frustrated spin ladder with alternating spin-1 and spin$\frac{1}{2}$ rungs. Phys. Rev. B, 81:024409, Jan 2010. doi: 10.1103/PhysRevB.81.024409. URL https://link.aps. org/doi/10.1103/PhysRevB.81.024409.

47 E. Dagotto, J. Riera, and D. Scalapino. Superconductivity in ladders and coupled planes. Phys. Rev. B, 45:5744-5747, Mar 1992. doi:10.1103/PhysRevB.45.5744. URL https: //link.aps.org/doi/10.1103/PhysRevB.45.5744.

48 M. Sigrist, T. M. Rice, and F. C. Zhang. Superconductivity in a quasi-one-dimensional spin liquid. Phys. Rev. B, 49: 12058-12061, May 1994. doi:10.1103/PhysRevB.49.12058. URL https://link.aps.org/doi/10.1103/PhysRevB . 49.12058.

49 Wang X. Haldane phase, impurity effects and spin ladders, volume 528. Springer, Berlin, Heidelberg, 1999. doi: https://doi.org/10.1007/BFb0106072. 